\title{
GENERALIZED ORDINAL SUMS AND THE DECIDABILITY OF BL-CHAINS
}

\author{
MICHAEL C. LASKOWSKI AND YVONNE V. SHASHOUA
}

\begin{abstract}
We present a general construction of a family of ordinal sums of a sequence of structures and prove an elimination theorem for the class of ordinal sums in an expanded language. From this we deduce the decidability of the class of $\Sigma$-ordinal sums of models of a decidable theory $T$. As an application of this result we prove that the theory of BL-chains is decidable.
\end{abstract}

\section{INTRODUCTION}

$B L$-chains, otherwise known as linearly ordered BL-algebras, were introduced by Hájek [Ha] to provide a general framework for proving completeness theorems in fuzzy logics. Traditionally, BL-chains are presented in a larger language, but it is fruitful to consider them as a class of ordered semigroups with additional properties that make the other operations definable (see Definition 4.2).

In this paper we begin by offering a very general definition of an ordinal sum of models of a theory (see Definition 2.3). This definition is general enough to include both the classical notion of an ordinal sum of relational structures given by Tarski $[\mathrm{Ta}]$ and analyzed by FefermanVaught [FV] and Fuchs' notion of an ordinal sum of ordered semigroups given in [Fu]. Our interest in this notion is that the classification of BLchains we give in [LS] says that the class of BL-chains is identical with the class of certain ordinal sums of basic forms (see Definitions 4.2 and 4.4 and Theorem 4.5). Thus, general theorems about classes of ordinal sums immediately give information about the class of BL-chains.

In Definition 2.3 we designate a family of $L$-structures to be ordinal sums of a sequence $\left\langle\mathcal{M}_{i}: i \in I\right\rangle$ of disjoint models of an $L$-theory $T$ indexed by a linear order $(I,<)$. All of these structures have universe $\amalg\left\{M_{i}: i \in I\right\}$ and each $\mathcal{M}_{i}$ is a a substructure of each of these structures. The ordinal sums differ in how the function and relation symbols are defined on tuples from the universe that are not contained in a single $M_{i}$. Any specific way of expanding a disjoint union to an

Date: June 2, 2003.

Partially supported by NSF Research Grant DMS 0071746. 
ordinal sum is an assignment $\Sigma$. We investigate the class of $\Sigma$-ordinal sums of models of $T$. Unfortunately, Example 2.4 demonstrates that the class of $\Sigma$-ordinal sums need not be an elementary class in the original language $L$. To remedy this, Definition 2.5 provides us with a canonical way of expanding and extending an ordinal sum to a larger language $L^{*}$. In this larger language the class of expanded $\Sigma$-ordinal sums of models of $T$ is elementary and is amenable to analysis.

Theorem 2.11, which is the main theorem of the paper, provides an elimination result for the class of expanded $\Sigma$-ordinal sums of models of $T$. Specifically, it asserts that every $L^{*}$-sentence is equivalent to a separated $L^{*}$-sentence (see Definition 2.10) modulo the theory of expanded $\Sigma$-ordinal sums of models of $T$. This theorem is similar to the elimination result given by Feferman-Vaught $[\mathrm{FV}]$, but there are two important improvements. First, as noted above, the theorem here holds for a much more general notion of an ordinal sum. Second, the expansion and extension of an ordinal sum mentioned above is much milder than what is done in Feferman-Vaught. That is, when Feferman and Vaught analyze ordinal sums they expand the structure to a language much richer than $L^{*}$. For our subsequent results it is desirable to have an elimination theorem with respect to the language $L^{*}$ as opposed to their richer language. In particular, Theorem 3.1, which asserts the decidability of the class of $\Sigma$-ordinal sums of models of $T$ whenever $T$ is decidable, follows by a straightforward reduction of the consistency of separated $L^{*}$-sentences to the consistency of colored linear orderings, which is known to be decidable by a theorem of Läuchli and Leonard [LL].

Finally, in Section 4 we use Theorem 3.1 to demonstrate that the elementary theory of BL-chains is decidable. Specifically, Theorem 3.1 reduces the decidability of the theory of BL-chains to the decidability of the theory of basic forms. However, Corollary 4.8 shows that the decidability of the theory of basic forms follows easily from the decidability of the theory of ordered abelian groups, which was established by Gurevich [Gur].

\section{AN ELIMINATION THEOREM FOR ORDINAL SUMS}

Definition 2.1. An ordered partition $\rho$ of $\langle 1, \ldots, n\rangle$ is a sequence $\left\langle s_{j}\right.$ : $j<t\rangle$, where each $s_{j}$ is a nonempty subsequence of $\langle 1, \ldots, n\rangle$ such that for each $1 \leq k \leq n, k$ occurs in exactly one $s_{j}$.

If $\rho=\left\langle s_{j}: j<t\right\rangle$ is an ordered partition of $\langle 1, \ldots, n\rangle$ and $\vec{a}$ is an $n$-tuple, then $s_{j}(\vec{a})$ is the subsequence $\left\langle a_{i}: i \in s_{j}\right\rangle$ of $\vec{a}$. 
If $\rho=\left\langle s_{j}: j<t\right\rangle$ is an ordered partition of $\langle 1, \ldots, n\rangle$ and $A=$ $\coprod\left\{A_{i}: i \in I\right\}$ is a disjoint union of sets indexed by a linear order $(I,<)$ then we say that $\vec{a} \in A^{n}$ induces $\rho$ if there are $i_{0}<i_{1}<\cdots<i_{t-1}$ from $I$ such that the elements of $s_{j}(\vec{a})$ are from $A_{i_{j}}$ for all $j<t$. As notation, we denote each $i_{j}$ by $i_{j}(\vec{a})$.

Definition 2.2. Let $T$ be any theory in the language $L$. A ( $k$-ary) $T$-definable function $h$ is a $(k+1)$-ary $L$-formula $\varphi_{h}(\vec{x}, y)$ such that $T \models \forall \vec{x} \exists ! y \varphi_{h}(\vec{x}, y)$. If a model $\mathcal{M}$ of $T$ is a substructure of an $L$ structure $\mathcal{N}$, then $h^{\mathcal{M}}(\vec{a})$ is the unique $b \in M$ such that $\mathcal{N} \models \varphi_{h}^{\mathcal{M}}(\vec{a}, b)$, where $\varphi_{h}^{\mathcal{M}}$ is the relativization of $\varphi_{h}$ to $M$.

Definition 2.3. Fix a language $L$ without constant symbols and fix an $L$-theory $T$. An $L$-structure $\mathcal{M}$ is an ordinal sum of models of $T$ if the universe of $M=\coprod\left\{M_{i}: i \in I\right\}$ is a disjoint union of universes of models $\mathcal{M}_{i}$ of $T$ and

- Each $\mathcal{M}_{i}$ is a substructure of $\mathcal{M}$;

- For each $n$-ary function symbol $f \in L$ and each ordered partition $\rho=\left\langle s_{j}: j<t\right\rangle$ of $\langle 1, \ldots, n\rangle$, there is a $k<t$ and a $T$-definable function $g$ such that

$$
f^{\mathcal{M}}(\vec{a})=g^{\mathcal{M}_{i_{k}(\vec{a})}}\left(s_{k}(\vec{a})\right)
$$

for all $\vec{a} \in M^{n}$ that induce $\rho$; and

- For each $n$-ary relation symbol $R \in L$ and each ordered partition $\rho=\left\langle s_{j}: j<t\right\rangle$ of $\langle 1, \ldots, n\rangle$, there is a sequence $\left\langle\delta_{j}\left(s_{j}(\vec{y})\right)\right.$ : $j<t\rangle$ of $L$-formulas and a truth function $\Phi:\{T, F\}^{t} \rightarrow\{T, F\}$ such that

$$
R^{\mathcal{M}}(\vec{a}) \leftrightarrow \Phi\left(W_{0}, \ldots, W_{t-1}\right)=T
$$

for all $\vec{a} \in M^{n}$ that induce $\rho$, where, for each $j<t, W_{j}=T$ if and only if $M_{i_{j}(\vec{a})} \models \delta_{j}\left(s_{j}(\vec{a})\right)$.

A mapping $\Sigma$ which sends function symbols of $L$ and ordered partitions to $T$-definable functions and sends relation symbols of $L$ and ordered partitions to sequences of $L$-formulas and truth functions is called an assignment. An ordinal sum of models of $T$ whose assignment is $\Sigma$ is called a $\Sigma$-ordinal sum of models of $T$.

The example below indicates that the class of $\Sigma$-ordinal sums of models of a theory need not be an elementary class of $L$-structures.

Example 2.4. Let $L=\{\leq\}$, let $T=T h(\omega, \leq)$, and let $\Sigma$ be the assignment making $\leq$ into a total order. Let $\mathcal{K}$ denote the class of $\Sigma$-ordinal sums of $T$. We will show that $\mathcal{K}$ is not elementary by finding elementarily equivalent $L$-structures $\mathcal{M}, \mathcal{N}$ with $\mathcal{M} \in \mathcal{K}$ and $\mathcal{N} \notin \mathcal{K}$. 
Let $\mathcal{M}$ be the $\Sigma$-ordinal sum generated from $\left\langle\mathcal{M}_{i}: i \in \mathbb{Q}\right\rangle$, where each $\mathcal{M}_{i}$ is isomorphic to $(\omega, \leq)$. Let $\mathcal{N}$ be the extension of $\mathcal{M}$ formed by adding a copy of $(\mathbb{Z}, \leq)$ inside a proper cut of $\mathbb{Q}$ (for definiteness say the cut of $\pi$ ). It is easily verified using Ehrenfeucht-Fraïssé games (in much the same way as one shows that $(\omega, \leq) \equiv\left(\omega^{\wedge} \mathbb{Z}, \leq\right)$ ) that $\mathcal{M} \equiv \mathcal{N}$. (In fact, $\mathcal{N}$ is an elementary extension of $\mathcal{M}$.) Now assume by way of contradiction that $\mathcal{N}$ were the $\Sigma$-ordinal sum of $\left\langle\mathcal{N}_{j}: j \in J\right\rangle$, where each $N_{j} \models T$. Choose $j \in J$ such that $N_{j} \cap \mathbb{Z} \neq \emptyset$. Since models of $T$ are closed under successors and predecessors, $\mathbb{Z} \subseteq N_{j}$. However, since every model of $T$ has a least element, some $M_{i} \subseteq N_{j}$ as well. However, since $N_{j}$ is convex, our construction guarantees that some other $M_{i^{\prime}} \subseteq N_{j}$ as well. Hence $N_{j}$ has more than one element with no immediate predecessor, which contradicts $\mathcal{N}_{j} \models T$.

In order to circumvent this example and to gain control of the elementary properties of the class, it is desirable to expand a $\Sigma$-ordinal sum of models of $T$ by adding new elements to represent the condensation classes under the natural equivalence relation of being elements of the same $\mathcal{M}_{i}$. As well, we add symbols to the language to make this equivalence relation explicitly definable. More formally, we have the following definition:

Definition 2.5. Fix a language $L$ without constant symbols. Let $L^{*}=L \cup\{V, \pi,<\}$, where $V$ is a new unary predicate, $\pi$ is a new unary function, and $<$ is a new binary relation. Associated to any representation $\mathcal{M}=\coprod\left\{\mathcal{M}_{i}: i \in I\right\}$ of an ordinal sum there is a canonical expansion of $\mathcal{M}$ to an $L^{*}$-structure $\mathcal{M}^{*}$ which is defined as follows:

- The universe of $M^{*}$ is $M \cup I$;

- The interpretation of $V\left(\mathcal{M}^{*}\right)$ is $M$;

- Symbols in $L$ are only interpreted on $V\left(\mathcal{M}^{*}\right)$, and their interpretation there is identical to their interpretation on $\mathcal{M}$;

- The symbol $<$ is only interpreted on $\neg V\left(\mathcal{M}^{*}\right)$ and its interpretation is $(I,<)$;

- The symbol $\pi$ is interpreted as a function from $V\left(\mathcal{M}^{*}\right)$ to $\neg V\left(\mathcal{M}^{*}\right)$, and $\pi^{\mathcal{M}^{*}}(a)=i$ if and only if $a \in M_{i}$.

It is clear that for any $L$-theory $T$ and any assignment $\Sigma$, the class of expanded $\Sigma$-ordinal sums of models of $T$ is an elementary class of $L^{*}$-structures.

We think of $L^{*}$ as being a '2-sorted' language and we will primarily concern ourselves with $L^{*}$-formulas whose variables 'know their sorts'. The following definition makes these notions precise. 
Definition 2.6. An $L^{*}$-formula $\varphi$ is 2-sorted if $\varphi \vdash V(x)$ or $\varphi \vdash \neg V(x)$ for every free variable $x$ occurring in $\varphi$. As notation, we typically denote the $V$-variables by $y$ 's and the $\neg V$-variables by $Z$ 's. The 2 sorted formulas $\varphi$ and $\psi$ are comparable if

$$
\varphi \vdash V(x) \text { if and only if } \psi \vdash V(x)
$$

for every variable $x$ occurring freely in both $\varphi$ and $\psi$. If $\varphi$ is 2 -sorted, then its 2-sorted negation $\neg^{\prime} \varphi$ is the 2-sorted $L^{*}$-formula that is comparable to $\varphi$ for which $\varphi\left(\mathcal{M}^{*}\right) \cap \neg \varphi\left(\mathcal{M}^{*}\right)=\emptyset$ for every $L^{*}$-structure $\mathcal{M}^{*}$. If $\varphi$ and $\psi$ are comparable 2-sorted formulas then the 2-sorted disjunction is defined to be $\neg^{\prime}\left(\neg^{\prime} \varphi \wedge \neg^{\prime} \psi\right)$. If $F$ is any set of comparable 2 -sorted formulas, then the set of 2-boolean combinations of $F$ is the closure of $F$ under conjunction and 2-sorted negation.

Our goal is to isolate a specific subset of the $L^{*}$-sentences, namely the set of separated $L^{*}$-sentences (see Definition 2.10) for which the dependence on the theory $T$ is separated from the dependence on the linear order $(I,<)$. To begin we give two variants of equivalence of formulas.

Definition 2.7. Let $T$ be an $L$-theory, let $\rho$ be an ordered partition of $\langle 1, \ldots, n\rangle$, and let $\Sigma$ be an assignment.

(1) Two comparable 2-sorted $L^{*}$-formulas $\gamma_{1}\left(y_{1}, \ldots, y_{n}, \vec{Z}\right)$ and $\gamma_{2}\left(y_{1}, \ldots, y_{n}, \vec{Z}\right)$ are $(\Sigma, \rho)$-equivalent if

$$
\mathcal{M}^{*} \models \gamma_{1}(\vec{a}, \vec{d}) \leftrightarrow \gamma_{2}(\vec{a}, \vec{d})
$$

for every expanded $\Sigma$-ordinal sum $\mathcal{M}^{*}$ of models of $T$, every $\vec{a} \in V\left(\mathcal{M}^{*}\right)^{n}$ that induces $\rho$ and for every $\vec{d} \in \neg V\left(\mathcal{M}^{*}\right)^{k}$.

(2) Two comparable 2-sorted $L^{*}$-formulas $\gamma_{1}\left(y_{1}, \ldots, y_{n}, \vec{Z}\right)$ and $\gamma_{2}\left(y_{1}, \ldots, y_{n}, \vec{Z}\right)$ are $\rho$-equivalent if

$$
\mathcal{M}^{*} \models \gamma_{1}(\vec{a}, \vec{d}) \leftrightarrow \gamma_{2}(\vec{a}, \vec{d})
$$

for every expanded ordinal sum $\mathcal{M}^{*}$ of models of $T$, every $\vec{a} \in$ $V\left(\mathcal{M}^{*}\right)^{n}$ that induces $\rho$ and for every $\vec{d} \in \neg V\left(\mathcal{M}^{*}\right)^{k}$.

Definition 2.8. For any nonempty set $S$ of variable symbols, let $\Gamma_{S}$ denote the 2-sorted $L^{*}$-formula $\bigwedge_{y, y^{\prime} \in S} V(y) \wedge \pi(y)=\pi\left(y^{\prime}\right)$.

We define a 2 -sorted $L^{*}$-formula $\delta_{S}^{*}$ for any $L$-formula whose free variables are among $S$ by induction on the complexity of $\delta$ as follows: For $\delta$ atomic, $\delta_{S}^{*}:=\delta \wedge \Gamma_{S} ;(\neg \delta)_{S}^{*}:=\neg \delta \wedge \Gamma_{S} ;(\delta \wedge \beta)_{S}^{*}:=\delta_{S}^{*} \wedge \beta_{S}^{*}$; and $(\exists y \delta)_{S}^{*}:=\exists y \delta_{S \cup\{y\}}^{*} \cdot$ 
If the $L$-formula $\delta\left(y_{1}, \ldots, y_{l}\right)$ has its free variables displayed (or more precisely if its free variables are among $\left.\left\{y_{1}, \ldots, y_{l}\right\}\right)$ we simplify notation and simply write $\delta^{*}\left(y_{1}, \ldots, y_{l}\right)$ in place of the more cumbersome $\delta_{\left\{y_{1}, \ldots, y_{l}\right\}}^{*}$.

For $\sigma$ any $L$-sentence and $Z$ any $(\neg V)$-variable symbol, let $\sigma^{Z}$ denote the 2 -sorted $L^{*}$-formula that represents the relativization of $\sigma$ to $\pi^{-1}(Z)$. That is,

- If $\sigma$ is $\exists w \delta(w)$ then $\sigma^{Z}:=\exists w\left(\pi(w)=Z \wedge \delta^{*}(w)\right)$;

- If $\sigma$ is $\forall w \delta(w)$, then $\sigma^{Z}:=\forall w\left(\pi(w)=Z \rightarrow \delta^{*}(w)\right)$.

The intuition is that $\delta^{*}\left(y_{1}, \ldots, y_{l}\right)$ or $\sigma^{Z}$ represents a finite piece of information about a specific condensation class, namely $\pi^{-1}\left(\pi\left(y_{1}\right)\right)$ or $\pi^{-1}(Z)$, respectively.

Definition 2.9. A separated $L^{*}$-formula is a 2 -sorted $L^{*}$-formula of the form $Q_{1} Z_{1} \ldots Q_{k} Z_{k} \eta\left(Z_{1}, \ldots, Z_{k}, Z_{k+1}, \ldots, Z_{m}\right)$, where each $Q_{i} Z_{i}$ is either $\exists Z_{i}$ or $\forall Z_{i}$ and $\eta$ is a 2-boolean combination of formulas of the form $Z_{i}<Z_{j}, Z_{i}=Z_{j}$ (which we call order atoms) and $L^{*}$-formulas of the form $\sigma^{Z_{i}}$, where $\sigma$ is a sentence of $L$.

One important aspect of being a separated $L^{*}$-formula is that it has no free $V$-variables. However, a typical separated $L^{*}$-formula may have unquantified $(\neg V)$ (i.e., $Z$ ) variables.

Definition 2.10. A separated $L^{*}$-sentence is a separated $L^{*}$-formula $Q_{1} Z_{1} \ldots Q_{k} Z_{k} \eta\left(Z_{1}, \ldots, Z_{k}\right)$, in which all $Z$-variables have been quantified out.

Theorem 2.11. Let $L$ be a language without constant symbols and let $T$ be any $L$-theory. For any assignment $\Sigma$ and any $L^{*}$-sentence $\sigma$ there is a separated $L^{*}$-sentence $\sigma_{\Sigma}$ such that $\mathcal{M}^{*} \models \sigma \leftrightarrow \sigma_{\Sigma}$ for every expanded $\Sigma$-ordinal sum $\mathcal{M}^{*}$ of models of $T$. Furthermore, given a recursive presentation of $L$, the mapping $(\Sigma, \sigma) \mapsto \sigma_{\Sigma}$ is recursive.

We will obtain this as a consequence of the more general result for formulas given in Proposition 2.19. Our strategy is to define, for each ordered partition $\rho$ of $\langle 1, \ldots, n\rangle$, a syntactically restrictive class of $\rho$ formulas. We then give a series of lemmas leading up to Proposition 2.19, which indicates that for any assignment $\Sigma$, the set of $\rho$ formulas contains a representative of every $(\Sigma, \rho)$-equivalence class of 2 -sorted $L^{*}$-formulas with free $V$-variables among $y_{1}, \ldots, y_{n}$.

Definition 2.12. Fix an ordered partition $\rho=\left\langle s_{i}: i<t\right\rangle$ of $\langle 1, \ldots, n\rangle$.

(1) A $\rho$-atom is any 2 -sorted $L^{*}$-formula of any of the following forms: 
- Order Atoms: $\pi\left(y_{j}\right)<Z_{k} ; Z_{j}<\pi\left(y_{j}\right) ; \pi\left(y_{j}\right)=Z_{k} ; Z_{j}<$ $Z_{k}$; or $Z_{j}=Z_{k} ;{ }^{1}$ or

- Delta Atoms: an $L^{*}$-formula $\delta^{*}\left(s_{i}(\vec{y})\right)$, corresponding to some $i<t$ and some $L$-formula $\delta\left(s_{i}(\vec{y})\right)$; or

- Sigma Atoms: an $L^{*}$-formula $\sigma^{Z_{j}}$ for some $L$-sentence $\sigma$.

(2) A $\rho$-conjunction is a finite conjunction of $\rho$-atoms.

(3) A quantifier-free $\rho$-formula is a 2-boolean combination of $\rho$ atoms.

(4) A $\rho$-formula has the form $Q_{1} Z_{1} \ldots Q_{l} Z_{l} \eta\left(y_{1}, \ldots, y_{n}, Z_{1}, \ldots, Z_{m}\right)$, where $l \leq m$ and each $Q_{j} Z_{j}$ is either $\exists Z_{j}$ or $\forall Z_{j}$, and $\eta$ is a quantifier-free $\rho$-formula.

Every $\rho$-formula is clearly 2 -sorted. Note that the 2 -sorted negation of a $\rho$-atom is $\rho$-equivalent to a finite disjunction of $\rho$-atoms. This observation is the key to the following lemma, which gives a 'standard form' for quantifier-free $\rho$-formulas that will be used later.

Lemma 2.13. For every ordered partition $\rho=\left\langle s_{i}: i<t\right\rangle$ of $\langle 1, \ldots, n\rangle$, every quantifier-free $\rho$-formula $\eta(\vec{y}, \vec{Z})$ is $\rho$-equivalent to a quantifierfree $\rho$-formula $\hat{\eta}$ of the form $\bigvee_{j<r} \theta_{j}$, where each $\theta_{j}$ is a $\rho$-conjunction. Moreover, we may assume that for every $j<r$ and $i<t, \theta_{j}$ contains exactly one delta atom whose free variables are among $s_{i}(\vec{y})$. Furthermore, the mapping $\eta \mapsto \hat{\eta}$ is recursive.

Proof: Given a quantifier-free $\rho$-formula $\eta$, first write $\eta$ in Disjunctive Normal Form (in the sense of Definition 2.6). Then eliminate 2 -sorted negations of $\rho$-atoms one at a time and use the distributive property of $\wedge$ over $\vee$, giving a formula $\hat{\eta}$ that is $\rho$-equivalent to $\eta$. Finally, by taking conjunctions of delta atoms from the same $s_{i}(\vec{y})$ and adding dummy delta atoms such as $\left(y_{k}=y_{k}\right)_{s_{i}(\vec{y})}^{*}$ as needed we obtain the requisite $\hat{\eta}$. This procedure is recursive.

The next lemma enumerates some routine operations under which the family of $\rho$-formulas is closed.

Lemma 2.14. For any $n$ and any ordered partition $\rho$ of $\langle 1, \ldots, n\rangle$, if $\gamma_{1}\left(y_{1}, \ldots, y_{n}, \vec{Z}\right)$ and $\gamma_{2}\left(y_{1}, \ldots, y_{n}, \vec{Z}\right)$ are comparable $\rho$-formulas, then each of the formulas $\neg^{\prime} \gamma_{1}, \gamma_{1} \wedge \gamma_{2}, \exists Z\left(\neg V(Z) \wedge \gamma_{1}\right)$ and $\forall Z(\neg V(Z) \rightarrow$ $\gamma_{1}$ ) are $\rho$-equivalent to $\rho$-formulas.

Proof: Suppose that the $\rho$-formulas $\gamma_{1}$ and $\gamma_{2}$ have the form $Q_{1} Z_{1} \ldots Q_{k} Z_{k} \eta_{1}\left(y_{1}, \ldots, y_{n}, \vec{Z}\right)$ and $Q_{1} W_{1} \ldots Q_{k^{\prime}} W_{k^{\prime}} \eta_{2}\left(y_{1}, \ldots, y_{n}, \vec{W}\right)$,

\footnotetext{
${ }^{1}$ Literally, each of these formulas should be conjoined with formulas specifying the sorts of each variable, e.g., $Z_{j}<Z_{k}$ should be $Z_{j}<Z_{k} \wedge \neg V\left(Z_{j}\right) \wedge \neg V\left(Z_{k}\right)$.
} 
respectively where $\eta_{1}$ and $\eta_{2}$ are quantifier-free $\rho$-formulas. We may assume that the sequences of variables $\vec{Z}$ and $\vec{W}$ are disjoint. Then $Q_{1}^{*} Z_{1} \ldots Q_{k}^{*} Z_{k} \neg^{\prime} \eta_{1}$ (where each $Q^{*}$ is the 'opposite quantifier' of $Q$ ) is $\rho$-equivalent to $\neg^{\prime} \gamma_{1}$, and is itself $\rho$-equivalent to a $\rho$-formula by the note preceding Lemma 2.13. Also,

$$
Q_{1} Z_{1} \ldots Q_{k} Z_{k} Q_{1} W_{1} \ldots Q_{k^{\prime}} W_{k^{\prime}}\left(\eta_{1} \wedge \eta_{2}\right)\left(y_{1}, \ldots, y_{n}, \vec{Z}, \vec{W}\right)
$$

is $\rho$-equivalent to $\gamma_{1} \wedge \gamma_{2}$. Closure of the set of $\rho$-formulas under the other two operations is immediate from their definition.

The main work in establishing Proposition 2.19 concerns quantification of a $V$-variable, which is the content of Lemma 2.16 below. How this quantification is handled depends both on the formula and on the ordered partition.

Definition 2.15. Suppose that $\rho=\left\langle s_{i}: i<t\right\rangle$ is an ordered partition of $\langle 1, \ldots, n\rangle$ and $k<n$. We say that $\rho$ links $n$ to $k$ if both $n$ and $k$ are elements of the same $s_{i}$. If $\rho \operatorname{links} n$ to $k$, then $\rho^{-}$is the ordered partition of $\langle 1, \ldots, n-1\rangle$ formed by replacing that $s_{i}$ in $\rho$ by $s_{i}^{\prime}$, which is formed by deleting $n$ from $s_{i}$. We say that $\rho$ isolates $n$ if some $s_{i}=\langle n\rangle$. In this case, we let $\rho^{-}$be the partition of $\langle 1, \ldots, n-1\rangle$ formed by deleting that $s_{i}$ from $\rho$.

Lemma 2.16. (1) For every $n$ and $k<n$, every ordered partition $\rho$ of $\langle 1, \ldots, n\rangle$ that links $n$ to $k$, and every $\rho$-formula $\gamma$ there is a $\rho^{-}$-formula $\hat{\gamma}$ that is $\rho^{-}$-equivalent to $\exists y_{n}\left(\gamma \wedge \pi\left(y_{n}\right)=\pi\left(y_{k}\right)\right)$.

(2) For every $n$, every ordered partition $\rho$ of $\langle 1, \ldots, n\rangle$ that isolates $n$, and every $\rho$-formula $\gamma$, there is a $\rho^{-}$-formula $\hat{\gamma}$ that is $\rho^{-}$equivalent to $\exists y_{n}\left(\gamma \wedge \bigwedge_{k<n} \pi\left(y_{n}\right) \neq \pi\left(y_{k}\right)\right)$.

In both cases the mapping $\gamma \mapsto \hat{\gamma}$ is recursive.

The following general lemma, a variant of which was suggested by the referee, unifies the proofs of the two cases.

Lemma 2.17. Let $\left\{\alpha_{j}: j<r\right\}$ and $\left\{\beta_{j}: j<r\right\}$ be $L^{*}$-formulas with no $Z$-variables occurring in any $\beta_{j}$. The formula $\exists y_{n} \vec{Q} \vec{Z} \bigvee_{j<r}\left(\alpha_{j} \wedge \beta_{j}\right)$ is equivalent to $\bigvee_{\emptyset \neq W \subseteq r} \psi_{W}$, where $\psi_{W}:=\exists y_{n}\left(\bigwedge_{j \in W} \beta_{j} \wedge \vec{Q} \vec{Z} \bigvee_{j \in W} \alpha_{j}\right)$. (Here $\vec{Q} \vec{Z}$ denotes quantification over any subset of the $Z$-variables.)

Proof: An easy induction on the quantifier length of $\vec{Q} \vec{Z}$ implies that $\vec{Q} \vec{Z} \bigvee_{j<r}\left(\alpha_{j} \wedge \beta_{j}\right)$ is equivalent to

$$
\bigvee_{\emptyset \neq W \subseteq r}\left(\bigwedge_{j \in W} \beta_{j} \wedge \vec{Q} \vec{Z} \bigvee_{j \in W} \alpha_{j}\right)
$$


(for left to right take $W=\left\{j<r: \beta_{j}\right.$ holds $\}$ ). The lemma follows since $\exists$ commutes with $\bigvee$.

Proof of Lemma 2.16 For both cases fix an ordered partition $\rho=$ $\left\langle s_{i}: i<t\right\rangle$ of $\langle 1, \ldots, n\rangle$ and a $\rho$-formula $\gamma$. As notation, suppose $n \in s_{i}$. By Lemma $2.13 \gamma$ is $\rho$-equivalent to a $\rho$-formula of the form $\vec{Q} \vec{Z} \bigvee_{j<r} \theta_{j}\left(y_{1}, \ldots, y_{n}, \vec{Z}\right)$, where each $\theta_{j}$ is a $\rho$-conjunction with exactly one delta atom $\delta_{j}^{*}\left(s_{i}(\vec{y})\right)$ with free variables among $s_{i}(\vec{y})$.

In Case (1) apply Lemma 2.17 with $\beta_{j}\left(s_{i}(\vec{y})\right):=\delta_{j}^{*}\left(s_{i}(\vec{y})\right)$ and $\alpha_{j}$ to be $\theta_{j}$ with the conjunct $\delta_{j}^{*}\left(s_{i}(\vec{y})\right)$ deleted and every occurrence of $\pi\left(y_{n}\right)$ replaced by $\pi\left(y_{k}\right)$. By Lemma 2.17 the formula $\exists y_{n} \vec{Q} \vec{Z} \bigvee_{j<r} \theta_{j}$ is equivalent to $\bigvee_{\emptyset \neq W \subseteq r} \psi_{W}$, where each $\psi_{W}$ has the form $\exists y_{n}\left(\bigwedge_{j \in W} \delta_{j}^{*}\left(s_{i}(\vec{y})\right) \wedge\right.$ $\left.\vec{Q} \vec{Z} \bigvee_{j \in W} \alpha_{j}\right)$ Since the variable $y_{n}$ does not occur in any $\alpha_{j}$, each $\psi_{W}$ is equivalent to

$$
\left(\exists y_{n} \bigwedge_{j \in W} \delta_{j}^{*}\left(s_{i}(\vec{y})\right)\right) \wedge \vec{Q} \vec{Z} \bigvee_{j \in W} \alpha_{j}
$$

But the first conjunct is simply the $\rho^{-}$-atom $\left(\exists y_{n} \bigwedge_{j \in W} \delta_{j}\left(s_{i}(\vec{y})\right)\right)^{*}$ and the second conjunct is a $\rho^{-}$-formula by Lemma 2.14. The lemma follows by another application of Lemma 2.14 .

In Case (2) we apply Lemma 2.17 taking $\beta_{j}:=\delta_{j}^{*}\left(y_{n}\right) \wedge \bigwedge_{k<n} \pi\left(y_{k}\right) \neq$ $\pi\left(y_{n}\right)$ and $\alpha_{j}$ to be $\theta_{j}$ with the conjunct $\delta_{j}^{*}\left(y_{n}\right)$ deleted. Then the formula $\exists y_{n}\left(\vec{Q} \vec{Z} \bigvee_{j<r} \theta_{j} \wedge \bigwedge_{k<n} \pi\left(y_{n}\right) \neq \pi\left(y_{k}\right)\right)$ is equivalent to $\bigvee_{\emptyset \neq W \subseteq r} \psi_{W}$, where each $\psi_{W}$ has the form

$$
\exists y_{n}\left(\bigwedge_{j \in W} \delta_{j}^{*}\left(y_{n}\right) \wedge \bigwedge_{k<n} \pi\left(y_{n}\right) \neq \pi\left(y_{k}\right) \wedge \vec{Q} \vec{Z} \bigvee_{j \in W} \alpha_{j}\right)
$$

Since $\rho$ isolates $n$, for every $W \exists y_{n} \bigwedge_{j \in W} \delta_{j}\left(y_{n}\right)$ is an $L$-sentence which we denote by $\sigma_{W}$. Additionally, the only occurrences of the variable $y_{n}$ in the $\alpha_{j}$ 's involve $\pi\left(y_{n}\right)$. Consequently, if we let $Z^{*}$ denote a new variable symbol that does not occur in $\gamma$, then each $\psi_{W}$ is $\rho^{-}$-equivalent to

$$
\exists Z^{*}\left(\neg V\left(Z^{*}\right) \wedge \sigma_{W}^{Z^{*}} \wedge \bigwedge_{k<n} Z^{*} \neq \pi\left(y_{k}\right) \wedge \vec{Q} \vec{Z} \bigvee_{j \in W} \hat{\alpha}_{j}\right)
$$

where $\hat{\alpha}_{j}$ is formed from $\alpha_{j}$ by replacing every occurrence of $\pi\left(y_{n}\right)$ by $Z^{*}$. Each $\hat{\alpha}_{j}$ is a $\rho^{-}$-formula since each $\alpha_{j}$ was a $\rho$-formula. So the lemma follows by applications of Lemma 2.14.

Lemma 2.18. For every assignment $\Sigma, n \in \omega$, and ordered partition $\rho$ of $\langle 1, \ldots, n\rangle$, every 2-sorted atomic $L^{*}$-formula $\varphi\left(y_{1}, \ldots, y_{n}, \vec{Z}\right)$ is 
$(\Sigma, \rho)$-equivalent to a quantifier-free $\rho$-formula $\eta\left(y_{1}, \ldots, y_{n}, \vec{Z}\right)$. Furthermore, the map $(\Sigma, \rho, \varphi) \mapsto \eta$ is recursive.

Proof: One first proves the following result for $L$-terms:

Claim 1. For every L-term $\tau\left(y_{1}, \ldots, y_{n}\right)$ and for every ordered partition $\rho=\left\langle s_{j}: j<t\right\rangle$ of $\langle 1, \ldots, n\rangle$, there is a $k<t$ and a $T$-definable function $h$ in the variables $s_{k}(\vec{y})$ such that

$$
\tau^{\mathcal{M}}(\vec{a})=h^{\mathcal{M}_{i_{k}(\vec{a})}}\left(s_{k}(\vec{a})\right)
$$

for all $\Sigma$-ordinal sums $\mathcal{M}$ of models of $T$ and for all $\vec{a}$ from $M$ that induce $\rho$.

Then, given this result for $L$-terms, one can read off the $\rho$-formula corresponding to each atomic $L$-formula from $\rho$ and the assignment $\Sigma$. Finding $\rho$-formulas that are $(\Sigma, \rho)$-equivalent to the other $L^{*}$-atomic formulas such as $V(\tau(\vec{y})), \pi(\tau(\vec{y}))=Z$ and $\pi\left(\tau_{1}(\vec{y})\right)<\pi\left(\tau_{2}(\vec{y})\right)$ is also routine.

We our now ready to prove our main elimination result. Note that since every $L^{*}$-sentence is trivially 2 -sorted, Theorem 2.11 follows as an immediate corollary to the following proposition.

Proposition 2.19. Let $L$ be a language without constant symbols and let $T$ be any L-theory. For any assignment $\Sigma$, for any 2 -sorted $L^{*}$ formula $\varphi\left(y_{1}, \ldots, y_{n}, \vec{Z}\right)$, and for every ordered partition $\rho$ of $\langle 1, \ldots, n\rangle$, there is a $\rho$-formula $\varphi_{\Sigma, \rho}$ that is $(\Sigma, \rho)$-equivalent to $\varphi$. Moreover, given a recursive presentation of $L$, the mapping $(\Sigma, \varphi, \rho) \mapsto \varphi_{\Sigma, \rho}$ is recursive.

Proof: Fix an assignment $\Sigma$. Call a 2-sorted $L^{*}$-formula explicitly 2-sorted if it is in the closure of the atomic 2-sorted $L^{*}$-formulas under the operations of conjunction, 2-sorted negation, $\exists y(V(y) \wedge \ldots)$ and $\exists Z(\neg V(Z) \wedge \ldots)$. A straightforward induction shows that every 2sorted formula is equivalent to an explicitly 2 -sorted formula. We argue that every explicitly 2 -sorted $L^{*}$-formula $\varphi\left(y_{1}, \ldots, y_{n}, \vec{Z}\right)$ satisfies the following condition: For every ordered partition $\rho$ of $\langle 1, \ldots, n\rangle$, there is a $\rho$-formula that is $(\Sigma, \rho)$-equivalent to $\varphi$.

That the condition holds for all atomic 2-sorted $L^{*}$-formulas is the content of Lemma 2.18. If $\varphi\left(y_{1}, \ldots, y_{n}, \vec{Z}\right)$ and $\psi\left(y_{1}, \ldots, y_{n}, \vec{Z}\right)$ are comparable explicitly 2 -sorted $L^{*}$-formulas that satisfy the condition, then $\varphi \wedge \psi, \neg^{\prime} \varphi$ and $\exists Z(\neg V(Z) \wedge \varphi)$ all satisfy the condition by Lemma 2.14. Thus, it suffices to prove that if the explicitly 2 -sorted $L^{*}$-formula $\varphi\left(y_{1}, \ldots, y_{n}, \vec{Z}\right)$ satisfies the condition, then for every ordered partition $\rho$ of $\langle 1, \ldots, n-1\rangle$ there is a $\rho$-formula $(\Sigma, \rho)$-equivalent to $\exists y_{n} \varphi$. 
So fix such a $\varphi$ and an ordered partition $\rho$ of $\langle 1, \ldots, n-1\rangle$. It follows from Lemma 2.14 that the formulas $\varphi_{k}:=\varphi \wedge \pi\left(y_{n}\right)=\pi\left(y_{k}\right)$ for $k<n$ and the formula $\varphi_{n}:=\varphi \wedge \bigwedge_{k<n} \pi\left(y_{n}\right) \neq \pi\left(y_{k}\right)$ also satisfy the condition. For each $k<n$ let $\rho_{k}$ be the (unique) ordered partition of $\langle 1, \ldots, n\rangle$ that extends $\rho$ and links $n$ to $k$ and let $\rho_{n}$ be the ordered partition of $\langle 1, \ldots, n\rangle$ that extends $\rho$ and isolates $n$. Note that $\left(\rho_{k}\right)^{-}=\rho$ for all

$k \leq n$. For each $k \leq n$ let $\gamma_{k}\left(y_{1}, \ldots, y_{n}, \vec{Z}\right)$ be a $\rho_{k}$-formula that is $\left(\Sigma, \rho_{k}\right)$-equivalent to $\varphi_{k}$. It is easily checked that the $\rho$-formula

$$
\bigvee_{k \leq n} \hat{\gamma}_{k}\left(y_{1}, \ldots, y_{n-1}, \vec{Z}\right)
$$

(where $\hat{\gamma}_{k}$ is generated from $\exists y_{n} \gamma_{k}$ using Lemma 2.16) is $(\Sigma, \rho)$-equivalent to $\exists y_{n} \varphi$.

The following corollary follows easily.

Corollary 2.20. Fix a language $L$ without constant symbols, an $L$ theory $T$, an assignment $\Sigma$, and sequences $\left\langle\mathcal{M}_{i}: i \in I\right\rangle$ and $\left\langle\mathcal{N}_{i}: i \in I\right\rangle$ of models of $T$, each indexed by the same linear order $(I,<)$, such that each $\mathcal{M}_{i}$ is L-elementarily equivalent to $\mathcal{N}_{i}$. Then the expanded $\Sigma$ ordinal sum generated by $\left\langle\mathcal{M}_{i}: i \in I\right\rangle$ is $L^{*}$-elementarily equivalent to the expanded $\Sigma$-ordinal sum generated by $\left\langle\mathcal{N}_{i}: i \in I\right\rangle$.

Proof: Let $\mathcal{M}^{*}$ and $\mathcal{N}^{*}$ be the expanded $\Sigma$-ordinal sums of models of $T$ generated by $\left\langle\mathcal{M}_{i}: i \in I\right\rangle$ and $\left\langle\mathcal{N}_{i}: i \in I\right\rangle$ respectively. Because of Theorem 2.11 it suffices to show that

$$
\mathcal{M}^{*} \models \gamma \quad \text { if and only if } \quad \mathcal{N}^{*} \models \gamma
$$

for all separated $L^{*}$-sentences. But this follows immediately since the underlying linear orders of $\mathcal{M}^{*}$ and $\mathcal{N}^{*}$ are identical and for every order atom $\sigma^{Z}, \mathcal{M}^{*} \models \sigma^{i}$ if and only if $\mathcal{N}^{*} \models \sigma^{i}$ for every $i \in I$.

\section{The DECIDABILITY of $\sum$-ORDINAL SUMS}

The following theorem is the goal of this section. By examining the proof it is clear that the assumption of a finite language can be eliminated if one instead assumes a recursive presentation of both the language and the assignment $\Sigma$.

Theorem 3.1. If $T$ is a decidable theory in a finite language without constant symbols, then for all assignments $\Sigma$, the elementary class of expanded $\Sigma$-ordinal sums of models of $T$ is decidable. Moreover, the decidability is uniform in $\Sigma$. 
In Corollary 3.6 we prove that the decidability of the class of $\Sigma$ ordinal sums of models of $T$ (in the original language $L$ ) follows from this.

Our proof of Theorem 3.1 reduces the decidability of the class of expanded $\Sigma$-ordinal sums of models of $T$ to the decidability of the class of colored linear orderings. To make this latter notion precise requires the following definition.

Definition 3.2. (1) For every $i \in \omega$, let $U_{i}$ be a unary predicate symbol. Let $L^{c}:=\{\leq\} \cup\left\{U_{i}: i \in \omega\right\}$, which we call the language of colored linear orders.

(2) Let $T_{\text {l.o. }}$ denote the usual theory of linear orders, (i.e., axioms that assert that $\leq$ is reflexive, transitive, and antisymmetric) in the language $L^{c}$.

(3) For $\tau$ any sentence of $L^{c}$, let $|\tau|=\max \left\{i \in \omega: U_{i}\right.$ occurs in $\tau\}+1$.

(4) For any $k \in \omega$, let $\Delta_{k}$ be the $L^{c}$-sentence:

$$
\forall x\left[\left(\bigvee_{j<k} U_{j}(x)\right) \wedge \bigwedge_{i<j<k} \neg\left(U_{i}(x) \wedge U_{j}(x)\right)\right] .
$$

(5) A $k$-colored linear order is an $L^{c}$-structure $\mathcal{D}$ such that $\mathcal{D} \models$ $T_{\text {l.o. }} \cup\left\{\Delta_{k}\right\}$.

The following theorem is due to Läuchli and Leonard [LL]. A proof of this result in the language of pure linear orders appears in [Ros]. However, as noted in the original paper of Läuchli and Leonard, there is no obstruction in extending the argument to the language $L^{c}$ (see e.g., Theorem A.6.10 of [Hod]).

Theorem 3.3. $T_{\text {l.o. }}$ is a decidable $L^{c}$-theory.

We will reduce the decidability of $\Sigma$-ordinal sums of models of $T$ to the set $A$ defined in the following corollary.

Corollary 3.4. Let $A:=\left\{\langle k,\ulcorner\sigma\urcorner\rangle: k \geq|\sigma|\right.$ and $\sigma$ is an $L^{c}$-sentence that holds in some $k$-colored linear order $\}$. Then $A$ is recursive.

Proof: This follows immediately from Theorem 3.3 since $\langle k,\ulcorner\sigma\urcorner\rangle \in$ $A$ if and only if $T_{l . o .} \forall \forall\left(\Delta_{k} \rightarrow \neg \sigma\right)$.

The following proposition specifies the reduction mentioned above.

Proposition 3.5. Let $L$ be a finite language without constant symbols. Fix a decidable $L$-theory $T$. For any separated $L^{*}$-sentence $\gamma$, there is an integer $k(\gamma) \in \omega$ and an $L^{c}$-sentence $h(\gamma)$ such that $|h(\gamma)| \leq k(\gamma)$ and the following two conditions hold: 
(1) If $\gamma$ is true in the expansion of some ordinal sum of models of $T$, then $h(\gamma)$ is true in some $k(\gamma)$-colored linear order.

(2) If $h(\gamma)$ holds in some $k(\gamma)$-colored linear order, then for every assignment $\Sigma, \gamma$ is true in some expanded $\Sigma$-ordinal sum of models of $T$.

Furthermore, both $k(\gamma)$ and $h(\gamma)$ can be obtained recursively from $\gamma$.

Proof: We will begin by giving the construction of the map $h$. Let $\gamma:=Q_{1} Z_{1} \ldots Q_{l} Z_{l} \eta\left(Z_{1}, \ldots, Z_{l}\right)$ be a separated $L^{*}$-sentence. We do the following five steps (note that each step can be performed recursively):

Step 1 List all $L$-sentences $\sigma_{0}, \ldots, \sigma_{m-1}$ such that for some $Z, \sigma^{Z}$ is a sigma atom occurring in $\eta$.

Step 2 Let $\pm \sigma$ denote either $\sigma$ or $\neg \sigma$. Using the list of $\sigma$ 's, form every possible combination of $\pm \sigma_{0} \wedge \pm \sigma_{2} \wedge \ldots \wedge \pm \sigma_{m-1}$, giving us a list of $2^{m}$ sentences $\psi_{1}, \ldots, \psi_{2^{m}}$.

Step 3 Remove from our list any $\psi_{n}$ 's that are inconsistent with $T$ and reindex, giving us a list $\left\{\psi_{0}, \ldots, \psi_{v-1}\right\}$ for some $v \leq 2^{m}$. Let $k(\gamma)$ denote this $v$. Since $T$ is decidable this step can be performed recursively.

Note that by construction our $\psi_{n}$ 's have the following two properties:

(a) For every $n<k(\gamma), \psi_{n}$ is consistent with $T$.

(b) For every $\mathcal{N} \models T$, there is exactly one $n<k(\gamma)$ such that $\mathcal{N} \models \psi_{n}$.

We also note that, for each $i<m, \sigma_{i}$ is equivalent (modulo $T$ ) to a disjunction $\bigvee_{n \in \Lambda_{i}} \psi_{n}$, where $n \in \Lambda_{i}$ if and only if $\sigma_{i}$ occurs positively in $\psi_{n}$.

Step 4 Let $\eta^{\prime}\left(Z_{1}, \ldots, Z_{l}\right)$ be the formula obtained from $\eta$ by replacing each occurrence of $\sigma_{i}$ by $\bigvee_{n \in \Lambda_{i}} \psi_{n}$. That is, $\sigma_{i}^{Z_{j}}$ becomes $\left(\bigvee_{n \in \Lambda_{i}}\left(\psi_{n}\right)^{Z_{j}}\right)$.

Step 5 Define $h(\gamma)$ to be the $L^{c}$-sentence $Q_{1} Z_{1} \ldots Q_{l} Z_{l} \eta^{*}\left(Z_{1}, \ldots, Z_{l}\right)$, where $\eta^{*}$ is obtained from $\eta^{\prime}$ by replacing each occurrence of $\psi_{n}^{Z_{l}}$ by $U_{n}\left(Z_{l}\right)$. Note that $|h(\gamma)| \leq k(\gamma)$ by construction.

Now we prove $(1)$. Let $\mathcal{M}:=\coprod\left\{\mathcal{M}_{i}: i \in I\right\}$ be a $\Sigma$-ordinal sum of models of $T$. Let $\mathcal{M}^{*}$ be the canonical expansion of $\mathcal{M}$ and assume that $\mathcal{M}^{*} \models \gamma$. We define a $k(\gamma)$-colored linear order $\mathcal{D}$ such that $\mathcal{D} \models h(\gamma)$. Let $\mathcal{D}:=\left\langle D,<,\left\{U_{n}: n \in \omega\right\}\right\rangle$ be defined by taking $(D,<)$ to be $(I,<)$ and interpreting each color $U_{n}$ as follows:

- If $n<k(\gamma)$, then for every $d \in D$, define $\mathcal{D} \models U_{n}(d)$ if and only if $\mathcal{M}^{*} \models \psi_{n}^{d}$ (and, using our definition of $\Sigma$ and induction, this holds if and only if $\mathcal{M}_{d} \models \psi_{n}$ ). 
- If $n \geq k(\gamma)$, then for every $d \in D$, define $\mathcal{D} \models \neg U_{n}(d)$ (or, equivalently, $\left.U_{n}^{\mathcal{D}}=\emptyset\right)$.

Note that $\mathcal{D} \models \Delta_{k(\gamma)}$ because the list of $\psi_{n}$ 's has property (b) mentioned in Step 3. It is readily checked that $\mathcal{D} \models h(\gamma)$.

Now we prove (2). Let $\mathcal{D}:=\left\langle D,<,\left\{U_{n}: n \in \omega\right\}\right\rangle$ be a $k(\gamma)$ colored linear order such that $\mathcal{D} \models h(\gamma)$. Fix an assignment $\Sigma$. We will construct a $\Sigma$-ordinal sum of models of $T, \mathcal{M}$ whose canonical expansion $\mathcal{M}^{*} \models \gamma$. Note that for each $d \in D$, there is a unique $n(d)<k(\gamma)$ such that $\mathcal{D} \models U_{n(d)}(d)$. As well, the sentence $\psi_{n(d)}$ is consistent with $T$. So choose a set $\left\{\mathcal{M}_{d}: d \in D\right\}$ of disjoint $L$ structures with $\mathcal{M}_{d} \models T \cup\left\{\psi_{d}\right\}$. Let $M=\coprod\left\{M_{d}: d \in \mathcal{D}\right\}$ be the universe of $\mathcal{M}$. It is easily verified that there is a unique way of defining the functions and relations on $\mathcal{M}$ so as to make $\mathcal{M}$ a $\Sigma$-ordinal sum of models of $T$. Let $\mathcal{M}^{*}$ be the canonical expansion of $\mathcal{M}$ as given in Definition 2.5. Then $\mathcal{M}^{*} \models \gamma$ and our proof is complete.

The proof of Theorem 3.1 is now immediate.

Proof of Theorem 3.1: Let $T$ be a decidable theory in a finite language without constant symbols and let $\Sigma$ be any assignment. Given an $L^{*}$-sentence $\sigma$, let $g_{\Sigma}(\sigma)$ be the separated $L^{*}$-sentence obtained from Theorem 2.11. It is easily verified that $\sigma$ is true in some $\Sigma$-ordinal sum of models of $T$ if and only if $\left\langle k\left(g_{\Sigma}(\sigma)\right),\left\ulcorner h\left(g_{\Sigma}(\sigma)\right)\right\urcorner\right\rangle \in A$. As the maps $h, g_{\Sigma}$ and the set $A$ are all recursive, this implies that the theory of expanded $\Sigma$-ordinal sums of models of $T$ is decidable.

As noted in Example 2.4 the class of $\Sigma$-ordinal sums of models of $T$ in the language $L$ need not be an elementary class. Despite this, we call the class decidable if the set of codes of $L$-sentences true in every $\Sigma$-ordinal sum of models of $T$ is recursive.

Corollary 3.6. Let $T$ be a decidable theory in a finite language $L$ without constant symbols. Then for any assignment $\Sigma$, the class of $\Sigma$-ordinal sums of models of $T$ is decidable.

Proof: Let $\sigma$ be any $L$-sentence. Then $\sigma^{V}$, the relativization of $\sigma$ to $V\left(\mathcal{M}^{*}\right)$ is an $L^{*}$-sentence and for any $\Sigma$-ordinal sum $\mathcal{M}^{*}$ of models of $T$, we have $\mathcal{M} \models \sigma$ if and only if $\mathcal{M}^{*} \models \sigma^{V}$ where $\mathcal{M}^{*}$ is the canonical expansion of $\mathcal{M}$. That is, the decidability of the $\Sigma$-ordinal sums of models of $T$ is reducible to the decidability of the expanded $\Sigma$-ordinal sums, so we are done by Theorem 3.1. 


\section{The DECIDABILITY OF THE THEORY OF BL-CHAINS}

$B L$-chains, otherwise known as linearly ordered BL-algebras, were introduced by Hájek [Ha] to provide a general framework for proving completeness theorems in fuzzy logics. Traditionally, BL-algebras are presented in the language $L_{B L}=\{*, \leq, \Rightarrow, \cup, \cap, 0,1\}$, but it turns out that each of the symbols is definable in any BL-chain restricted to the sublanguage $L=\{*, \leq\}$, so we work in this smaller language. Throughout this section, with the only exception being the proof of Lemma 4.8, the underlying language is taken to be $L=\{*, \leq\}$. In [LS] we offer a characterization of the class of BL-chains which we proceed to summarize.

Definition 4.1. An ordered abelian semigroup $(S, *, \leq)$ is a linear order $\leq$ and a commutative and associative operation $*$ which satisfies

$$
x \leq y \quad \text { implies } \quad x * z \leq y * z
$$

for all $x, y, z \in S$. An ordered abelian group $(G, *, \leq)$ is an ordered abelian semigroup with an identity element such that every element has an inverse. In other words, $(G, *)$ is a group.

The definition of a BL-chain given below is readily seen to be equivalent to the definition in the language $L_{B L}$ given by Hájek in [Ha]. Specifically, the other constants and operations of $L_{B L}$ can be interpreted as follows: 1 denotes the top element of the chain and 0 denotes the bottom; $x \cap y=\min \{x, y\} ; x \cup y=\max \{x, y\}$; and $x \Rightarrow y=1$ whenever $x \leq y$, while $x \Rightarrow y=$ the largest $z$ such that $x * z=y$ whenever $y \leq x$.

Definition 4.2. A $B L$-chain is an $L$-structure $(A, *, \leq)$ satisfying the following axioms:

(1) The relation $\leq$ is a linear order on $A$ with a top and a bottom element;

(2) $(A, *, \leq)$ is an ordered abelian semigroup;

(3) The top element is the identity element of $(A, *)$; and

(4) For all $y \leq x$ there is a largest $z$ such that $x * z=y$.

In [LS] we obtain an algebraic characterization of the class of BLchains that is easily stated in terms of ordinal sums. To enable us to state it we require two more definitions.

Definition 4.3. Let $(G, *, \leq)$ be any ordered abelian group.

(1) The negative cone of $G$ is the substructure $(N(G), *, \leq)$ of $G$ with universe $\left\{x \in G: x<0_{G}\right\}$. 
(2) The extended negative cone $\left(N_{-\infty}(G), *, \leq\right)$ is an extension of $N(G)$ with universe $N(G) \cup\{-\infty\}$, where the definitions of $*$ and $\leq$ are extended so as to satisfy $\forall x(x *(-\infty)=(-\infty) * x=$ $(-\infty))$ and $\forall x(-\infty \leq x)$.

(3) Let $d \in N(G)$. The truncation of $N(G)$ at $d$ is the structure $T(G, d)$ with universe $\{x \in N(G): x \geq d\}$, where the operation $*_{T}$ is defined by:

$$
x *_{T} y:= \begin{cases}x *_{G} y & \text { if } x *_{G} y>d \\ d & \text { if } x *_{G} y \leq d\end{cases}
$$

and the relation $\leq_{T}$ is inherited from $G$.

Definition 4.4. A basic form is any ordered abelian semigroup $(S, *, \leq)$ that satisfies one of the following four conditions:

(1) $S$ is a singleton;

(2) $(S, *, \leq)$ is isomorphic to a negative cone of an ordered abelian group;

(3) $(S, *, \leq)$ is isomorphic to an extended negative cone of an ordered abelian group; or

(4) $(S, *, \leq)$ is isomorphic to a truncation $T(G, d)$ of an ordered abelian group.

In Lemma 4.6 below we will see below that the class of basic forms is an elementary class. As notation, let $T_{b . f}$. denote its theory. The following theorem is the content of Theorem 3.5 of [LS].

Theorem 4.5. Let $\Sigma$ is the assignment that makes $\leq$ a total order on an ordinal sum of models of $T_{b . f}$. and defines $x * y=\min \{x, y\}$ whenever $x$ and $y$ are from different $M_{i}$ 's. Then the class of BL-chains is precisely the class of $\Sigma$-ordinal sums of models $\left\langle\mathcal{M}_{i}: i \in I\right\rangle$ of $T_{b . f}$. such that $(I,<)$ has a first element $i(0)$, a last element $i(*), \mathcal{M}_{i(0)}$ has $a \leq$-least element, and $\mathcal{M}_{i(*)}$ is a singleton.

In order to get our decidability result we need to show that the class of basic forms is decidable. As a prelude to this, we show that the class is elementary.

Lemma 4.6. The class of basic forms is an elementary class. In fact, its theory $T_{b . f .}$ is finitely axiomatizable.

Proof: It follows from results in [LS] that each of the classes of singletons, negative cones, extended negative cones, and truncations can be axiomatized by a single sentence. For the class of singletons this is clear. Lemma 2.3 of [LS] (which is similar to Proposition 1, page 154 of $[\mathrm{Fu}])$ states that an ordered abelian semigroup $(S, *, \leq)$ is 
a negative cone of an ordered abelian group if and only if it satisfies the following two elementary properties:

(1) For all $x, y \in S, x>x * y$ and

(2) For each $x, y \in S$ such that $x>y$, there is a unique $z$ such that $x * z=y$.

Similarly, an ordered abelian semigroup $(S, *, \leq)$ is an extended negative cone of an ordered abelian group if and only if it has a least element $d$ and $(S \backslash\{d\}, *, \leq)$ is a negative cone. Finally, Lemma 2.4 of $[\mathrm{LS}]$ states that an ordered abelian semigroup $(S, *, \leq)$ with a least element $d$ is a truncation of an ordered abelian group if and only if the following four elementary properties hold:

(1) For any $x, y \in S, x * y \leq x$.

(2) For any $x, y \in S$, if $x * y=x$, then $x=d$.

(3) There are $x, y \in S, x, y>d$ such that $x * y=d$.

(4) For each $x, y \in S$ such that $x>y$, there is a largest $z$ such that $x * z=y$.

Thus, the class of basic forms is axiomatized by the sentence $\sigma^{S i} \mathrm{~V}$ $\sigma^{N C} \vee \sigma^{E N C} \vee \sigma^{T r}$, where the four sentences axiomatize the classes of singletons, negative cones, extended negative cones, and truncations, respectively.

The following theorem is due to Gurevich [Gur] and is at the heart of the proof of Corollary 4.8 .

Theorem 4.7. The theory of ordered abelian groups is decidable.

Corollary 4.8. The theory of basic forms is decidable.

Proof: It suffices to show that each of the four basic forms is decidable. Clearly, the theory of the singleton is decidable as there is only one one-element ordered abelian semigroup up to isomorphism.

The theory of negative cones is decidable since the negative elements of an ordered abelian group are definable.

The demonstration that the theory of extended negative cones is decidable follows easily, since an extended negative cone is isomorphic to a negative cone once its minimal element is removed.

To see that the theory of truncations is decidable requires some manipulations of languages. First, let $L_{T}=\left\{*_{T}, \leq\right\}$, where $*_{T}$ is a new binary function symbol and let $\sigma_{T}^{T r}$ be identical to $\sigma^{T r}$, except that every occurrence of $*$ in $\sigma^{T r}$ is replaced by $*_{T}$ in $\sigma_{T}^{T r}$. It clearly suffices to show that the set of (codes of) $L_{T}$ consequences of $\sigma_{T}{ }^{T r}$ is recursive. 
Next, let $L_{1}=\{*, \leq, 0, d\}$. Since the $L$-theory $T_{\text {o.a.g. }}$ of ordered abelian groups is decidable,

$$
T_{1}:=T_{\text {o.a.g. }} \cup\{\forall x(x * 0=x)\} \cup\{d<0\}
$$

is a decidable $L_{1}$-theory. Let $\delta(x):=d \leq x<0$ and let $L_{2}=$ $L_{1} \cup\left\{*_{T}\right\}$. Let $\Gamma$ be the sentence defining the operation $*_{T}$ given in Definition 4.3(3) for any truncation $T(G, d)$. More precisely, for all $x, y$ realizing $\delta, x *_{T} y=x * y$ whenever $x * y>d$, and $x *_{T} y=d$ whenever $x * y \leq d$. It follows that $T_{2}=T_{1} \cup\{\Gamma\}$ is a decidable $L_{2}$-theory.

But now, it follows from Lemma 2.4 of [LS] that for every truncation $\left(D, *_{T}, \leq\right) \models \sigma_{T}^{T r}$ there is an $L_{2}$-structure $\left(G, *, \leq, 0, d, *_{T}\right) \models T_{2}$ with $D=\delta(G)$. Conversely, if $\left(G, *, \leq, 0, d, *_{T}\right) \models T_{2}$, then $\left(\delta(G), *_{T}, \leq\right.$ )$\models \sigma_{T}^{T r}$. It follows that for every $L_{T}$-sentence $\sigma, \sigma$ is consistent with the $L_{T}$-theory of truncations if and only if $\sigma^{\delta}$, the relativization of $\sigma$ to $\delta(G)$, is consistent with $T_{2}$. As the latter theory is decidable, the decidability of the theory of truncations follows.

Theorem 4.9. The theory of BL-chains is decidable.

Proof: It follows immediately from Corollaries 3.6 and 4.8 that the theory of $\Sigma$-ordinal sums of models of $T_{b . f}$. is decidable, where $\Sigma$ is the assignment given in Definition 4.2. But the theory of BL-chains is clearly a finite extension of the theory of $\Sigma$-ordinal sums of models of $T_{b . f .}$, so it is decidable as well.

\section{REFERENCES}

[FV] S. Feferman and R.L. Vaught, The first order properties of products of algebraic systems, Fundamenta Mathematicae, 47(1959), 57-103.

[Fu] L. Fuchs, Partially Ordered Algebraic Systems, Pergamon Press, New York, 1963.

[Gur] Y. Gurevich, Elementary properties of ordered Abelian groups, American Mathematical Society Translations, 46(1965), 165-192.

[Ha] P. Hájek, Metamathematics of Fuzzy Logic, Kluwer Academic Publishers, Boston, 1998.

[Hod] W. Hodges, Model Theory, Cambridge University Press, Cambridge, 1993.

[LL] H. Läuchli and J. Leonard, On the elementary theory of linear order, Fundamenta Mathematicae, 59(1966) 109-116.

[LS] M.C. Laskowski, and Y.V. Shashoua, A classification of BL-algebras, Fuzzy Sets and Systems, 131(2002), no. 3, 271-282.

[Ros] J. Rosenstein, Linear Orderings, Academic Press, New York, 1982.

[Ta] A. Tarski, Ordinal Algebras, North Holland, Amsterdam, 1956. 
Department of Mathematics, University of Maryland, College Park, MD 20742

E-mail address: mcl@math.umd.edu

Department of Mathematics, University of Maryland, College Park, MD 20742 